parait grandement désirable que tous les intéressés se mettent d'accord d'abord sur une définition pratique de l'acidité des caséines industrielles, ensuite sur l'adoption d'une ou plusieurs méthodes standard pour donner l'acidité.

Je suggère que dans ce but il soit fait appel: d'une part aux producteurs de caséine, et en particulier à leurs deux principaux groupements qui sont l'Union de Caséineries coopératives des Charentes et du Poitou à Surgères, et le Syndicat des industriels fabricants de dérivés et sousproduits du lait à Paris ;

d'autre part aux fabricants de matières plastiques et de colle tant français qu'étrangers, ces derniers étant mêmes les plus importants au point de vue du tonnage de la caséine employée ;

enfin aux chimistes susceptibles de répondre aux desiderata des industriels pour la mise au point de méthodes d'analyse adéquates.

\title{
AU SUJET DE L'ACIDITÉ DES CASÉINES INDUSTRIELLES
}

$$
\text { par J.-A. QUOST. }
$$

Si l'on fait le dosage elassique de l'acidité d'un lait généralement de la même façon dans toutes les laiteries ou les laboratoires, ce qui rend les résultats comparables, il n'en est pas de même du dosage de l'acidité d'une caséine.

Chacun à sa méthode, chacun a ses résultats ; résultats qui ne signifient rien en eux-mêmes pour une même méthode et qui ne sont absolument pas comparables avec ceux des autres méthodes.

J'ai employé quelques-uns de ces procédés pour doser l'acidité d'un même échantillon de caséine présure. Les résultats allaient de 0,05 à $2 \%$ en acide lactique !

La question de la grosseur des grains n'intervenait pas. J'avais une même mouture très fine qui passait pour les $2 / 3$ au tamis de 70 , le reste au tamis 50.

Toutes les méthodes se classent en deux groupes :

$1^{\circ}$ Les méthodes par titrage sur filtrats d'épuisements qui consistent à traiter la caséine par des lavages à l'eau froide ou chaude ou à l'ébullition, pendant des temps donnés (et très variés) avec des répétitions données (et très varié $\mathrm{s}$ aussi), et à titrer les filtrats.

$\mathrm{Ou}$ bien on extrait presque rien (et ceci dépend pour une même mouture de la texture du grain, de la fabrication de la caséiné, etc...) parce que l'eau d'épuisement ne pénètre pas assez le grain; ou bien on extrait une bonne partie de l'acide lactique, mais on hydrolyse en même temps les matières albuminoïdes et l'on en trouve dans les filtrats d'extraction avant que l'acide lactique soit complètement éliminé de la masse. Ce que . j'ai pu constater. 
Les résultats sont done faux parce que dans le premier cas on n'a pas toute l'acidité lactique ; dans le deuxième cas parce que cette acidité lactique (si on l'a toute extraite et l'on n'en sait rien) s'augmente de celle propre aux produits d'hydrolyse. Il n'y a dès lors aucune raison pour s'arrêter à un temps ou à un nombre d'épuisements fixes. On peut aller ainsi jusqu'à complète extraction de l'acide lactique, après quoi on trouvera encore qu'une nouvelle eau d'épuisement est acide. L'expérience m'a montré en effet des eaux d'épuisements privés d'acide lactique qui donnaient une réaction acide importante correspondant à $0,05 \%$ de la caséine.

$2^{\circ}$ Les méthodes par titrage direct que j'ai dénommées ainsi parce que l'on ajoute dans ce cas peu à peu de la soude titrée à un mélange de caséine et d'eau chaude jusqu'à ce que la 'phtaléine du phénol additionnée au mélange ne se décolore plus après quelques temps d'agitation et de chauffage.

Ici, on a bien toute l'acidité lactique puisque la easéine est désagrégée, mais on a titré les groupements $\mathrm{CO}^{2} \mathrm{H}$. Or il n'y a aucun intérêt à les titrer puisque la caséine n'est pas vendue sous cet état dégradé et que cet état dégradé ne correspond à rien, ear là encore, on peut continuer l'hydrolyse; le moment ou la phtaléine du. phénol ne se décolore plus étant arbitraire. En effet si l'on continue à chauffer, de nouveau le mélange est acide. A la vérité, l'hydrolyse se fait alors pluslentementet le mélange reste plus longtemps coloré en rose. C'est pourquoi il semble qu'il y ait un point fixe. Que l'on opère à froid (ce sera plus long) à chaud $\left(60^{\circ}, 80^{\circ}\right.$ ou $\left.100^{\circ}\right)$ on arrive aux mêmes constatations.

La caséine est assez dégradée pour qu'une partie soit solubilisée au point de donner sur filtrat clair et limpide, provenant du traitement de la masse par l'alcool acétique, précipité et coloration par le réactif de Millon.

D'ailleurs en opérant par additions succesives de soude jusqu'au rose pour chaque addition, le mélange caséine + eau est constamment alcalin (milieu bien propre à l'attaque) parce que la phtaléine du phénol ne vire au rose que pour un $\mathrm{pH}$ se trouvant nettement en zonealcaline.

$\mathrm{Ce} \mathrm{pH}$ pour lequel vire la phtaléine du phénol correspond au point de salification des groupements $\mathrm{CO}^{3} \mathrm{H}$. La salification complète de l'acide lactique ne correspond pas à ce $\mathrm{pH}$, mais à celui plus acide pour lequel vire la paranitrophénol (jaune en liqueur alcaline, incolore en liqueur acide).

Et cette question de dosage de l'acidité lactique devient une question d'indieateur. Si nous prenons la paranitrophénol non influencé par l'acidité ou par l'alcalinité de la matière albuminoïde, nous pourrons doser l'acidité lactique seul. A la vérité, le virage du paranitrophénol vis-à-vis des acides tel que le lactique est malaisé à saisir. Mais si l'on renforce 
l'indicateur je veux dire si l'on masque la zone pour laquelle la décoloration du jaune est lentement progressive, on obtient un virage net. J'ai employé pour cela le bleu de méthylène et l'on a un virage du vert (alcalin) au bleu ciel (acide).

Pour avoir l'acide lastique occlus dans la masse on doit dissoudre la caséine, comme pour un dosage de matière grasse. Or on ne peut ici le faire avec un aside. Il faudrait un aside su'fisamment fort et, qui en tou cas, dézomposerait le lactique. Il faut donc avoir recours à la soude. Comme pour dégrader (ou dissoudre) cette caséine, il faudra être en zone al zaline, nous reviendrons en arrière par un titrage en retour avec un acide.

Et je propose une méthode ou plutôt une modification à des méthodes déjà existantes :

10 grammes de caséine finement moulue sont portés avec $100 \mathrm{~cm}^{3}$ d'eau distillée à l'ébullition.

On additionne peu à peu 20 ou $25 \mathrm{~cm}^{3}$ exactement mesurés de soude Dornic jusqu'à ce que la masse forme un liquide visqueụx (à chaud) ne présentant plus de grain en suspension.

On fait tomber $\mathrm{X}$ gouttes de solution de paranitrophénol dans l'éther a 20 grammes par litre et 5 gouttes de solution de bleu de méthyl ne à 0,5 par litre. L'ensemble est vert.

On ajoute jusqu'à virage au bleu un acide dont on connaitra la correspondance avec la soude Dornic (si l'on prend l'acide chlorydrique on le règlera à 4,05 pour un litre, de façon qu'il y ait correspondance exacte : $1 \mathrm{~cm}^{3}$ de l'un pour $1 \mathrm{~cm}^{3}$ de l'autre).

Soit $\mathrm{N}$ le nombre de $\mathrm{cm}^{3}$ de soude Dornic ajoutée, $\mathrm{n}$ le nombre de $\mathrm{cm}^{3}$ d'acide.

$$
\frac{(\mathrm{N}-\mathrm{n})}{10}=\mathrm{x} \mathrm{g} \text {. d'acide lactique } \% \text {. }
$$

\title{
L'INFLUENCE DU LAB-FERIMENT SUR LA MATURATION DU FROMAGE. EXPÉRIENCES AVEC LE BACKSTEIN RUSSE.
}

\author{
par J. HAWESSON, Agronome en Laiterie \\ (Travail de l'Institut de Laiterie de Wologda, Russie du Nord)
}

(Suite)

Ainsi, dans le travail ci-devant cité, ORLA-JENSEN admettait l'action de la pepsine même en milieu alsalin au tournesol. Plus tard (1906), se fondant sur les expériences faites par lui et mal réussies -ur l'accélération de la maturation du fromage $\mathrm{d}^{\mathbf{E}} \mathrm{Emmenthal,} \mathrm{au} \mathrm{moyen} 1$ addition de la pepsine, ainsi que sur les expériences déjà mentionnées de Spar LAN. zani et Bertozzi avec le Parmesan (1905), ORLA-JENSEn a brusquen'ent changé son opinion en refusant d'attribuer un rôle à. la pepsine, du moins dans la fabrication du fromage d'Emmenthal, en expliquant cela par 